\title{
Digressive Proportionality in a Cost Sharing of a Common Infrastructure Development
}

\author{
Izabela Heppner ${ }^{1}$ and Radosław Rudek ${ }^{2}$ \\ ${ }^{1}$ Wrocław University of Economics, \\ Komandorska 118/120, 53-345 Wrocław, Poland \\ ${ }^{2}$ General Tadeusz Kościuszko Military University of Land Forces \\ Czajkowskiego 109, 51-147 Wrocław, Poland
}

\begin{abstract}
The problem of a fair distribution is considered in relation to many areas and phenomena. As one of the oldest social problems, many methods of division have been proposed over the years. The most deeply rooted in the theory of justice are proportional divisions. However, they may be perceived as unfair for some practical cases. Let us consider a cost sharing of a natural gas infrastructure development. The main part of the cost is covered by an investor, whereas the rest, i.e. the total connection fee, has to be paid by customers willing to be connected to the pipeline. Since the revenue comes from consumption fees, therefore, the investment can start if the total expected gas consumption volume reaches a certain limit. If the total connection fee is divided proportionally among the customers based on their consumption volumes, then some of them will suffer from financial burden. On the other hand, connection fees of customers with small consumption will be marginal, but they will also use the common infrastructure. To prevent such cost sharing, which can be perceived as unfair, we will use the degressive proportionality principle. However, its practical application to determine a solution requires additional criteria and algorithms. We propose a cost sharing method derived from mathematical programming which optimizes a cost criterion based on a proportional distribution at the same time holding degressively proportional principle. Our proposal can be a compromise between different concepts of justice used in the cost sharing of a common infrastructure development.
\end{abstract}

Keywords: common infrastructure development; cost sharing; degressive proportionality; mathematical programming; proportional distribution 